\title{
The structural reliability analysis using explicit neural state functions
}

\author{
Agnieszka Dudzik ${ }^{1, *}$ and Beata Potrzeszcz-Sut ${ }^{1}$ \\ ${ }^{1}$ Kielce University of Technology, Faculty of Civil Engineering and Architecture, Avenue Tysiąclecia P.P. 7, Poland
}

\begin{abstract}
The present study considers the problems of stability and reliability of spatial truss susceptible to stability loss from the condition of node snapping. In the reliability analysis of structure, uncertain parameters, such us load magnitudes, cross-sectional area, modulus of elasticity are represented by random variables. Random variables are not correlated. The criterion of structural failure is expressed by the condition of non-exceeding the admissible load multiplier. In the performed analyses explicit form of the random variables function were used. To formulate explicit limit state functions the neural networks is used. In the paper only the time independent component reliability analysis problems are considered. The NUMPRESS software, created at the IFTR PAS, was used in the reliability analysis. The Hasofer-Lind index in conjunction with transformation method in the FORM was used as a reliability measure. The primary research method is the FORM method. In order to verify the correctness of the calculation SORM and Monte Carlo methods are used. The values of reliability index for different descriptions of mathematical model of the structure were determined. The sensitivity of reliability index to the random variables is defined.
\end{abstract}

\section{Introduction}

Component dimensions, environmental factors, material properties, and external loads are design variables. They may be characterized with statistical modes. The deterministic approach seeks out and defines a worst case or an extreme value to meet in the design. The probabilistic approach utilizes the statistical characterization and attempts to provide a desired reliability in the design. The deterministic approach introduces conservatism by specifying a factor of safety to cover unknowns. The probabilistic approach depends on the statistical characterization of a variable to determine its magnitude and frequency. The amount of data (how well the variable is defined) influences its extreme values. The studies proposed to treat building structure reliability as a random event and to analyse it with probability calculus methods. On the basis of these concepts, a semi-probabilistic method of limit states, using nowadays in Eurocodes, was developed.

The most advanced reliability analysis methods are probabilistic methods. Nowadays, the structural reliability theory is already a well-established research area. One can mention a number of textbooks and monographs, the most well-known being [1-5]. Special attention should be paid to publications by Harr [6], Nowak and Collins [7]. They present the basic concepts of reliability theory with a particular reference to their uses in civil engineering.

Interesting works are $[8,9]$ where numerical aspects of application of first order reliability method FORM in node snapping truss structures are considered. The same problem is considered in $[10,11]$. Different reliability assessment of structure propose work [12] about system reliability using serial and parallel systems. In order to determine the reliability of this approach it is necessary to set KAFM (kinematic admissible failure mechanism). The KAFM by spectral analysis of stiffness matrix are specified [13]. Reliability issues are important not only in static analysis, but also in stability analysis or dynamic analysis [14].

During the last three decades, neural network (NN) algorithms have been widely developed and used as a universal function approximator $[15,16] . \mathrm{NN}$ is a computational mechanism that is able to "acquire, represent, and compute a mapping from multivariate space of information to another, given a set of data representing that mapping" [17]. NN is capable of learning from training examples and finding meaningful solutions without the need to specify the relationship among variables. It can capture nonlinear and complex interactions among variables in a system [18]. A feedforward multilayer perceptrons (MLP) network was developed as an approximate limit state function [19]. Goh and Kulhawy [20] used MLP approach to model the limit state surface for reliability analysis. Deng et al. [21] described why and how to employ MLP technique to approximate the implicit performance functions and derivatives in FORM, SORM and MCS reliability analysis. Artificial neural networks in those works were multilayer perceptrons. 
MLP is the classic, most commonly used network structure in engineering issues. It is formed by neurons arranged in layers with one direction of signal flow. In the first layer (input) does not proceed in the computation processes. The structure of such a network, can be saved by the scheme: $X-H_{1}-H_{2}-\ldots-Y$, where: $X$ - number of inputs, $H_{1}, H_{2}, \ldots$ - the number of neurons in the next hidden layers, $Y$ - number of outputs. In most engineering applications it is sufficient to use only a single hidden layer and the use of two or more hidden layers is recommended when approximation discontinuous function. A mathematical description of flowing signals in these networks is relatively simple and it can be expressed in the explicit form by algebraic type of functional depending.

\subsection{Neural networks and reliability analysis}

Current research trends highlight the importance of structural reliability analysis methodologies able to provide improved estimates of the failure probability without an excessive increase in computational cost when compared with ordinary analyses methods. One of the new directions are hybrid methods of reliability analysis which using neural networks belonging to the group of soft computational methods.

Due to the way NN is used, three main concepts can be single out:

1. In FORM and SORM approximation methods, neural networks can replace polynomial functions of the limit state. This approach is presented, among others in the works [21, 22].

2. SN are used as so-called expert system. The learning and testing set of the network is the direct values of the probability of failure for the corresponding set values of the task parameters. An example of such an approach is presented in the paper [23].

3. FEM-based programs are used to compute patterns served learn a neural network. The $\mathrm{NN}$ is then used to generate samples used in the probabilistic analysis of structural reliability by Monte Carlo simulation. This approach is presented, e.g. in works [24, 25].

In the presented work, the first type of approach was used to analyse the reliability of a steel spatial truss. The course of action in the applied hybrid approach presents block diagram (Fig 1).

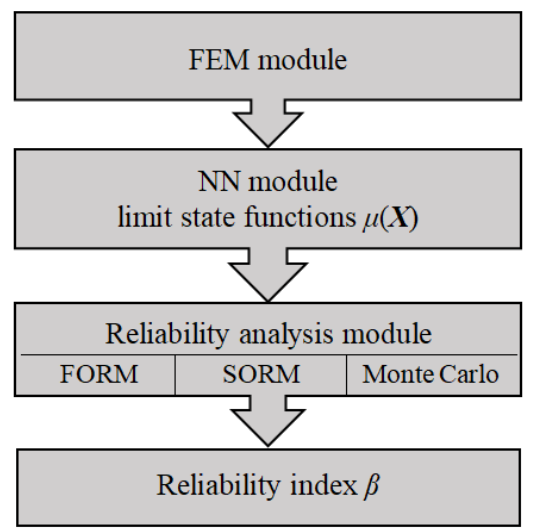

Fig. 1. Algorithm of applied approach to reliability analysis.
After defining the problem, that is accepting random variables, the FEM simulation is performed which allows to generate a learning and testing data set of the NN. After the simulation is completed, the network formulation phase follows. The prepared $\mathrm{NN}$ is then used to generate explicit state functions. For this purpose, the author's original MATLAB code programme was used.

Next step the form of explicit state functions are implemented in reliability software. This article aims to present the using of NUMPRESS software. That program was developed at the Institute of Fundamental Technological Research of the Polish Academy of Sciences by Kowalczyk, Rojek, Stocki, Bednarek, Tauzowski, Lasota, Lumelskyy, Wawrzyk [26].

The first step of the reliability analysis task using that software is a definition of the stochastic model. The user must provide marginal distributions of the basic random variables, for correlated variables, their correlation matrix (in NUMPRESS, an approximation joint probability density function is built by means of the socalled Nataf model). In the current version of the program, the following marginal distributions are available: exponential, Frechet, Gumbel, log-normal, normal, Rayleigh, uniform, Weibull and a general empirical distribution described by a set of experimental points.

After defining the variables, the limit state function is defined. In program, the limit state function is symbolically given in the standard math notation as a function of the basic random variables

The consecutive steps are dedicated to the selection, parameter setting and execution of the reliability analysis algorithm. The most computationally efficient methods for failure probability estimation are based on an approximation of the failure domain in the standard normal space. In FORM, the first order reliability method, the failure domain is approximated by the half space that is defined using the limit state surface linearized in the so-called design point. In the standard normal space, the design point is the point on the limit state surface which is closest to the origin. Finding a design point is a task for non-linear programming with limitations. There are two standard, gradient based algorithms for solving this problem implemented in NUMPRESS.

\subsection{Probabilistic methods}

The practical methods for reliability calculations can be broadly divided into approximate analytical methods like FORM (First Order Reliability Method) and SORM (Second Order Reliability Method) simulation based methods, for example, Importance Sampling and Monte Carlo. In each case the efficiency and applicability of a particular methodology largely depends on the efficient computation of the so-called design point. The design point and the 'region' around it contains the most important information regarding the probability of failure of structure. The calculation of the design point requires the solution of a constrained optimization 
problem. The concept of localization design point rely on realization of random variables from the failure surface which corresponds to the greatest value of the probability density function. With the linearization of the limit state function at the design point, it is possible to obtain a measure of reliability which is invariant due to the equivalent formulations of the boundary condition, i.e. the so-called Hasofer-Lind reliability index [27]. In the reliability analysis of a complex engineering structure a very large number of the system parameters can be considered to be random variables. The work of Hasofer and Lind, published in 1974, is recognized as the first important step towards the contemporary methods that make it possible to effectively and accurately estimate structural safety.

The basic method used in the study was FORM, which is one of approximation methods. The so-called Hasofer-Lind reliability index $\beta$ was adopted as a reliability measure. A great advantage of the FORM method is that it allows computing the sensitivity of the reliability index to a change in arbitrary parameters that are found in the problem description practically without additional computations. The sensitivity of the reliability index is computed as a first derivative of $\beta$ index with respect to a specified variable.

\section{Numerical analysis}

The numerical analysis presents application of the reliability FORM method to the analysis of the node snapping of spatial truss shown in Figure 2.

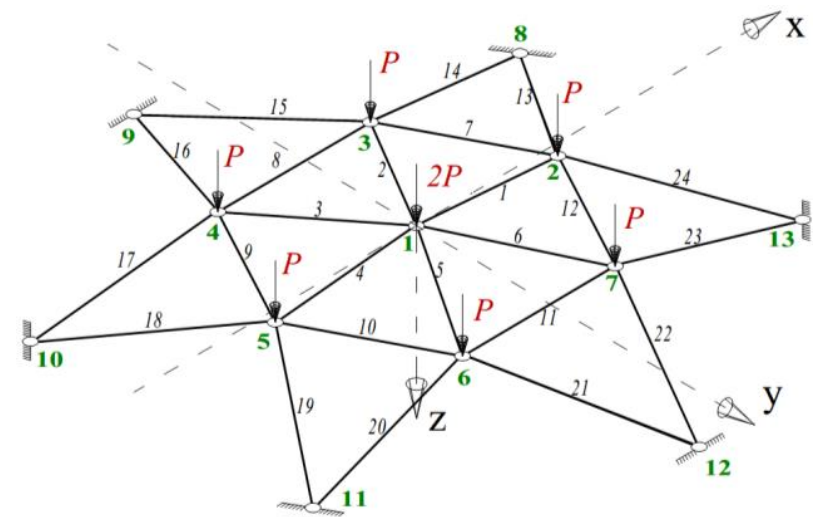

\begin{tabular}{|l|r|r|r|r|r|r|r|r|r|r|r|r|r|}
\hline No. Node & $\mathbf{1}$ & $\mathbf{2}$ & $\mathbf{3}$ & $\mathbf{4}$ & $\mathbf{5}$ & $\mathbf{6}$ & $\mathbf{7}$ & $\mathbf{8}$ & $\mathbf{9}$ & $\mathbf{1 0}$ & $\mathbf{1 1}$ & $\mathbf{1 2}$ & $\mathbf{1 3}$ \\
\hline
\end{tabular} \begin{tabular}{|l|r|r|r|r|r|r|r|r|r|r|r|r|r|r|}
\hline $\mathbf{x}[\mathbf{m}]$ & 0,0 & 25,00 & 12,50 & $-12,50$ & $-25,00$ & $-12,50$ & 12,50 & 43,30 & 0,00 & $-43,30$ & $-43,30$ & 0,00 & 43,30 \\
\hline
\end{tabular} \begin{tabular}{|l|l|l|l|l|l|l|l|l|l|l|l|l|l|l|}
$\mathbf{y}[\mathbf{m}]$ & 0,0 & 0,00 & $-21,65$ & $-21,65$ & 0,00 & 21,65 & 21,65 & $-25,00$ & $-50,00$ & $-25,00$ & 25,00 & 50,00 & 25,00 \\
\hline
\end{tabular}

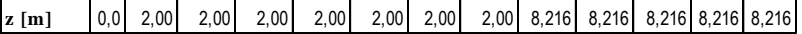
Fig. 2. Geometry of truss structure.

All elements were made of S235 steel with the yield point $f_{\mathrm{y}}=235 \mathrm{MPa}$ and modulus of elasticity $E=205 \mathrm{GPa}$. The assumption that the moment of the node snapping will never be preceded by the buckling of individual bars of the structure was also validated. Identification of the proper way of stability loss is the starting point for the selection of appropriate limit state function in reliability analysis. In connection with the above, the load bearing condition due to buckling behaviour for the most straining rod at the time of the node snapping was checked. Equilibrium path is presented in Figure 3.

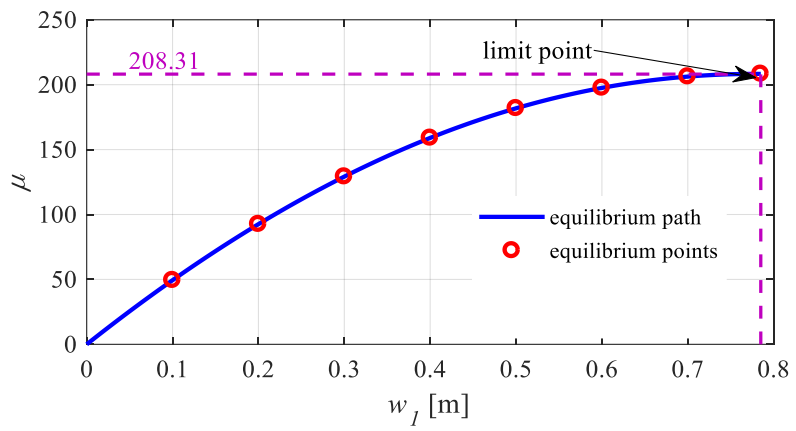

Fig. 3. Equilibrium path of the bar structure.

To determine the points on the equilibrium path, the Newton-Raphson algorithm and controlled by load was used In order to accurately determine the position of the limit point on the equilibrium path, the length of the step was determined adaptively [28]. Consequently, coordinates of the limit point: $w_{1}=0.785 \mathrm{~cm}, \mu=208.31$ were determined. On the basis of these coordinates, the limit function as the condition of the non-exceeding of the admissible vertical load multiplier of nodes was formulated:

$$
g(\mathbf{X})=1-\mu(\mathbf{X}) / 208.31
$$

where:

$\mu(\mathbf{X})$ - function of random variables describing formula for the load multiplier, while approaching to the limit point,

$\mathbf{X}=\{P, E, A\}-$ vector of random variables.

In this paper to formulate explicit state functions at every marked equilibrium point (see Fig. 3) a feedforward layered neural network with back-propagation of errors was used. In total, eight explicit formulas were formulated, i.e. eight neural networks were designed. In the learning process the pseudo-Gaussian LevenbergMarquardt method was used, which in certain cases is the fastest learning algorithm of feed-forward network. The learning process of the network has been verified by the mean squared error - MSE. In the testing process it was also checked for the average and the largest percentage error (avr $e p_{\mathrm{T}}$ and $\max e p_{\mathrm{T}}$ ) and the linear correlation coefficient $-r_{\mathrm{T}}$. The neural computations were made in the MATLAB environment using a package of Neural Network Toolbox library.

For the networks which performs mapping $\mu_{\mathrm{FEM}} \rightarrow \mu_{\mathrm{NN}}$ the following items of input $-\mathbf{x}$, target $-\mathbf{t}$ and output $-\mathbf{y}$ vectors were adopted: $\mathbf{x}_{(3 \times 1)}=\{P, E, A\}$, $\mathbf{t}_{(1 \times 1)}=\left\{\mu_{\mathrm{FEM}}\right\}, \mathbf{y}_{(1 \times 1)}=\left\{\mu_{\mathrm{NN}}\right\}$, where: $\mu_{\mathrm{FEM}}, \mu_{\mathrm{NN}}-$ load parameter computed by FEM or by NN.

Table 1. Features of task parameters.

\begin{tabular}{ccc}
\hline Parameter & Range of values & Scaling factor \\
\hline$P$ & $<0.7,1.3>[\mathrm{kN}]$ & $S_{\mathrm{P}}=1.365$ \\
\hline$E$ & $<143.5,266.5>[\mathrm{GPa}]$ & $S_{\mathrm{E}}=279825$ \\
\hline$A$ & $<0.0034,0.0064>\left[\mathrm{m}^{2}\right]$ & $S_{\mathrm{A}}=0.0067$ \\
\hline$\mu$ & $\begin{array}{c}\text { dependent on the } \\
\text { equilibrium point }\end{array}$ & $\begin{array}{c}\mathrm{S}_{\mu} \text { dependent on the } \\
\text { equilibrium point }\end{array}$ \\
\hline
\end{tabular}


The initial MLP architectures was formulated on the basis of preliminary computations, in which 64 sets of patterns were used for every equilibrium point (Fig. 3). Table 1 shows the ranges of values of the learning parameters and value of scaling factor for which trained neural networks for each of the point could be applied. For learning the network 43 random pattern data were used, whereas the other 21 patterns were used in the test. In the later numerical analysis MLP with one hidden layer model: $X-H-Y$ were applied. In order to optimally adjust the number of neurons in the $H$ layer the results were analysed for the several network structures.

In preparing the networks it was aimed to make these structures the simplest as possible, which determines the form of the approximation formula according to the rule: small network $\rightarrow$ simple approximation formula. It turned out that for the analysed issue, 5 neurons in the hidden layer are the optimal number. Consequently, to formulate explicit state functions at every equilibrium point the 3-5-1 structure of the network was selected. Maximum relative percentages errors for learning and testing then not exceed $1 \%$.

The output from the network, that is dependence $\mu_{\mathrm{NN}}(P, E, A)(2)$, can be formulated explicitly, through the known values of the network parameters:

$$
\begin{aligned}
& \mu_{N N}(E, A, P)= \\
& S_{\mu} \cdot\left(\mathbf{w}_{\mathbf{Y H}} \cdot\left(\tanh \left(\mathbf{w}_{\mathbf{H X}} \cdot\left[\begin{array}{lll}
\frac{E}{S_{E}} & \frac{A}{S_{A}} & \frac{P}{S_{P}}
\end{array}\right]^{\mathrm{T}}+\mathbf{b}_{\mathbf{H}}\right)\right)+\mathbf{b}_{\mathbf{Y}}\right)
\end{aligned}
$$

where:

$\mathbf{W}_{\mathbf{Y H}}$ - the weight of input $(X)$ and hidden $(H)$ layers connections,

$\mathbf{w}_{\mathbf{Y H}}-$ the weight of output $(Y)$ and $H$ layers connections, $\mathbf{b}_{\mathbf{H}}, \mathbf{b}_{\mathbf{Y}}-$ biases of neurons of subsequent layers of the network.

Network parameters (weight and biases), are most often calculated iteratively in the learning process.

After a decomposition the formula on $\mu_{\mathrm{NN}}$ (2) for the vertical displacement of the truss top node, which is $w_{1}=0.7 \mathrm{~m}$ can be represented as, for example:

$\mu_{\mathrm{NN}}=13339 \cdot \tanh \left(136.41 A+1.62 \mathrm{e}^{-4} E-1.32 P-1.26\right)$

$+6238.8 \cdot \tanh \left(209.93 A+4.52 \mathrm{e}^{-5} E-0.19 P-1.29\right)$

$+5778.7 \cdot \tanh \left(-217.82 A+8.31 \mathrm{e}^{-5} E+0.17 P+1.34\right)$

$+0.3912 \cdot \tanh \left(2005.4 A-9.70 \mathrm{e}^{-2} E-45.67 P+50.01\right)$

$+20007 \cdot \tanh \left(-130.54 A+1.44 \mathrm{e}^{-4} E+1.19 P+1.45\right)-6251.5$

In reliability analysis the following variables are used: load of nodes 1-7 $(P)$, modulus of elasticity $(E)$, cross-sectional area $(A)$, which are not correlated. Description of random variables is shown in Table 2.

Table 2. Description of the random variables.

\begin{tabular}{ccccc}
\hline $\begin{array}{c}\text { Random } \\
\text { variable }\end{array}$ & $\begin{array}{c}\text { Probability } \\
\text { density } \\
\text { function }\end{array}$ & $\begin{array}{c}\text { Mean } \\
\text { value }\end{array}$ & $\begin{array}{c}\text { Standard } \\
\text { deviation }\end{array}$ & $\begin{array}{c}\text { Coefficient } \\
\text { of variation }\end{array}$ \\
\hline$P$ & Normal & $1 \mathrm{kN}$ & $0.1 \mathrm{kN}$ & $10 \%$ \\
$E$ & Normal & $205 \mathrm{GPa}$ & $10.25 \mathrm{kN}$ & $5 \%$ \\
$A$ & Normal & $49 \cdot 10^{-4} \mathrm{~m}^{2}$ & $49 \cdot 10^{-6} \mathrm{~m}^{2}$ & $1 \%$ \\
\hline
\end{tabular}

The value of the Hasofer-Lind reliability index was determined with the FORM method, and for the sake of comparison, with SORM and Monte Carlo method. In reliability analysis the NUMPRESS software was used. Changing the value of reliability index $\beta$, while approaching to the limit point, is shown in Table 3 .

Table 3. Values of Hasofer-Lind reliability index.

\begin{tabular}{cccc}
\hline \multirow{2}{*}{$\begin{array}{c}\text { Load multiplier } \\
(\mu)\end{array}$} & \multicolumn{3}{c}{ Reliability index $(\beta)$} \\
\cline { 2 - 4 } & FORM & SORM & $\begin{array}{c}\text { Monte } \\
\text { Carlo }\end{array}$ \\
\hline 49.4 & 8.64 & 8.64 & 8.75 \\
\hline 92.6 & 5.64 & 5.63 & 5.82 \\
\hline 129.2 & 3.63 & 3.63 & 3.71 \\
\hline 159.4 & 2.18 & 2.17 & 2.21 \\
\hline 181.8 & 1.15 & 1.15 & 1.16 \\
\hline 199.8 & 0.35 & 0.34 & 0.37 \\
\hline 205.7 & 0.09 & 0.09 & 0.09 \\
\hline
\end{tabular}

The relative error of reliability index was estimated with the assumption that the reference is the Monte Carlo method. Differences between FORM/SORM and Monte Carlo method result of a number of sample size (it is equal 10000). The maximum relative error compare to Monte Carlo method amounted to $3.2 \%$. It means that the FORM method is good enough. Further analysis will be conducted using only the FORM method. Numerical results effects of changes on Hasofer-Lind reliability index value for different descriptions of the computational model are shown in Figure 4.

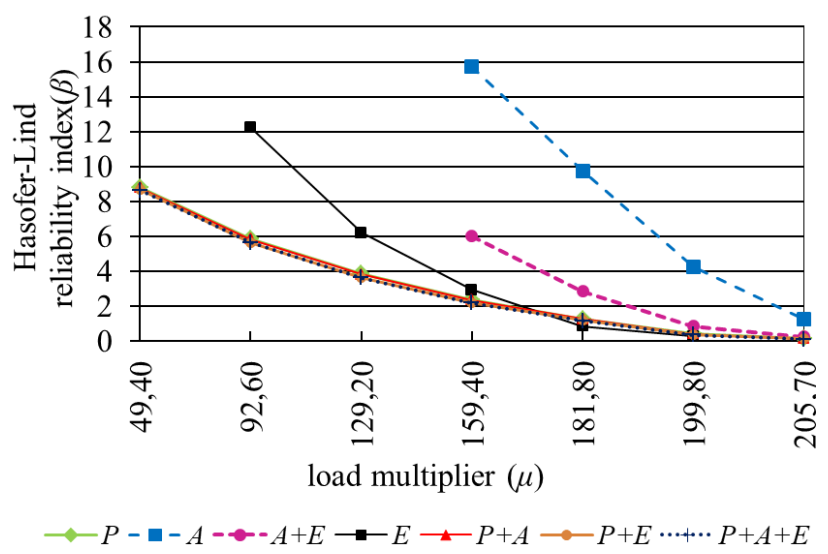

Fig. 4. Effect of the computational model description on the reliability index value.

Accounting for a larger number of random variables considerably reduces the reliability index value (Figure 4). Besides we can observe that the graphs for computational model description with random variable $P$ (cases: $P, P+A, P+E, P+A+E$ ) almost overlap. It means that random variable $P$ has highest impact on the value of reliability index.

Therefore, the next stage was sensitivity analysis. The reliability analysis performed with the NUMPRESS software also provides information on the sensitivity of reliability index to random variables and their 
characteristics of the probability density function (Figure 5).

a)

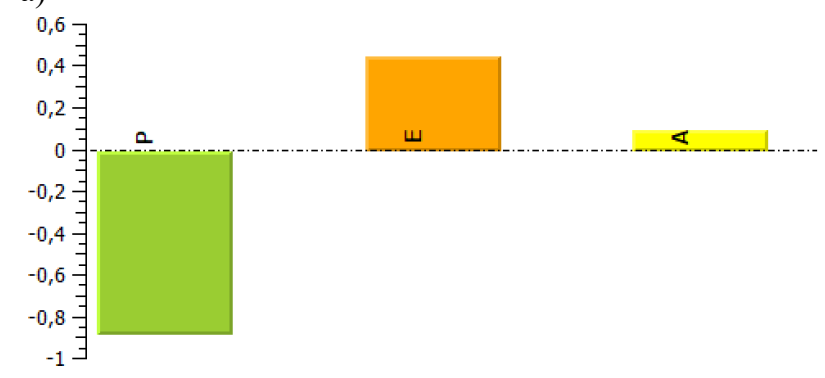

b)

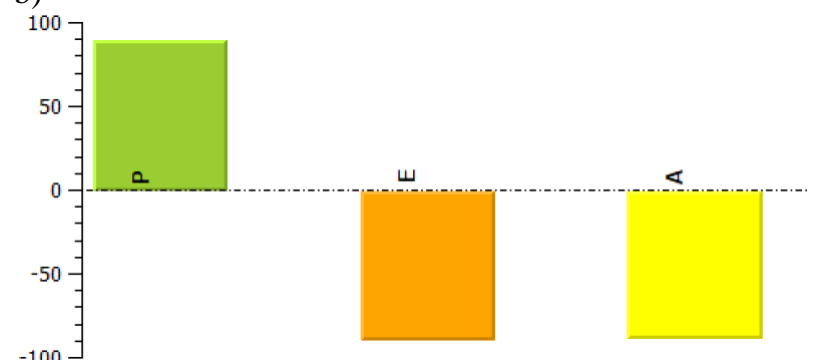

c)

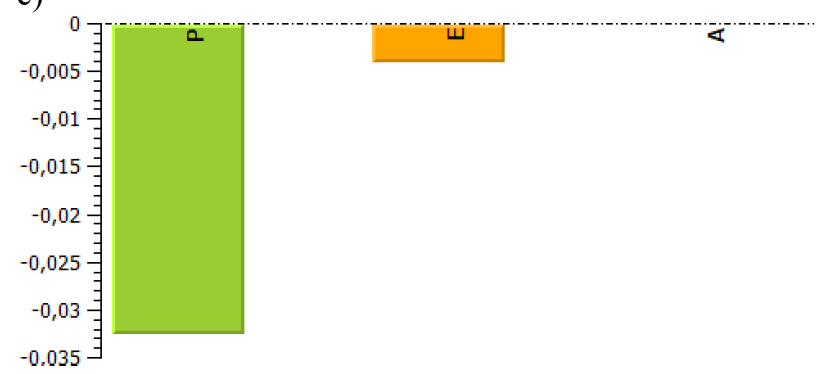

Fig. 5. Sensitivity of reliability index $\beta$ on: a) random variables, b) mean value of random variable, c) standard deviation of random variable.

The graphs (Figure 5) show which random variables have the highest impact on the value of reliability index. In that case the highest influence on values of reliability index has random variable $P$ which describes load in nodes. This conclusion also applies to the parameters of random variable distributions. We can also conclude (Figure 5c) that the value of the coefficient of variation of the random variable $P$ can have a significant impact on the value of the reliability index. The next part of analysis the effect of the assumed level of the variation coefficient of random variable $P$ on value of the reliability index was determined. For this purpose, five cases were further analysed. The description of the random variables from Table 1 was taken, except the level of the random variable of $P$. For these random variable the following values were assumed: $v_{\mathrm{p}}=\{2 \%$, $6 \%, 10 \%, 14 \%, 18 \%\}$. The results of the analysis in Figure 6 are shown.

Based on the tests, we can see that a change of the coefficient of variation compared to the output values $\left(v_{\mathrm{p}}=10 \%\right)$ generates significant changes in the reliability index (Figure 6). For example, for load multiplier $\mu=129.2$ and the coefficient of variation $\nu_{\mathrm{P}}=10 \%$, the reliability index is $\beta=3.63$, and for $\nu_{\mathrm{P}}=2 \%$ the value of the index increases by $176 \%(\beta=10.01)$, whereas for $\nu_{\mathrm{P}}=18 \%$ value of the index decreases by $27 \%(\beta=2.66)$.

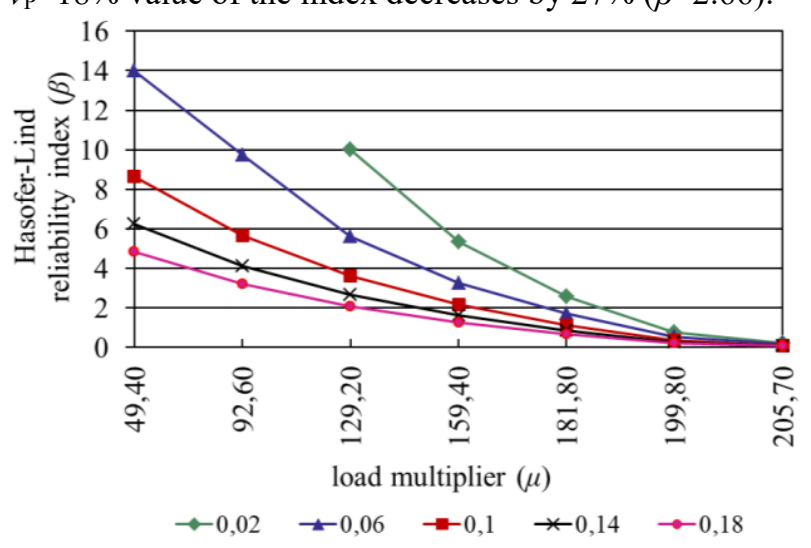

Fig. 6. Effect of the assumed level of the coefficient of variation for random variable $P$ on value of the reliability index.

These analysis show how important in reliability analysis is appropriate level of the coefficient of variation especially those random variables which impact on the value of reliability index is greatest.

\section{Conclusions}

The analysis of the results demonstrates that the FORM method is sufficiently precise and authoritative research method. The results indicate that the FORM method allows obtaining a quick response, which makes it possible to use the method in engineering practice as one of the modules of computational software that support structure design. We observed significant differences in the values of reliability index determined by the FORM method for different descriptions of the mathematical model. We observed that the lowest values of reliability index are for case where we have the highest number of variables $(P+A+E)$. Accounting for a larger number of random variables extends the computation time, yet such a manner of formulating the problem makes it possible to give a more accurate evaluation of a structure safety. In the case the $\mathrm{NN}$ is used to generate explicit state functions, the calculation time, especially for Monte Carlo method, is much shorter than the calculations performed with the use of implicit state function (from almost $1 \mathrm{~h}$ to $1 \mathrm{~min},[11])$.

Sensitivity analysis is an important element in the assessment of the impact of random variables on the reliability index value and thus on the factors which determine the safety of the structure. If the reliability index sensitivity due to the random variable $X_{i}$ is low when compared with other variables, it can be stated that the impact of this variable on failure probability is small, and in successive computations it can be treated as a deterministic parameter. Besides we should carefully to take level of the coefficient of variation especially those random variables which impact on the value of reliability index is greatest. So level of the coefficient of variation should be taken on the basis of statistical studies of the building strength and buildings materials and products. 
However, it has to be remembered at all times that the FORM method yields the best results when only one design point exists, the limit state function is not strongly linear and it is differentiable. The most commonly encountered disadvantage, also observed by other users, involves problems with calculating the gradients of the limit state function. Consequently, before taking a decision on applying the method to other problems in the structure analysis, it is necessary to run a number of functionality tests.

Besides in those performed studies, it has been demonstrated that neural networks are suitable for explicit formulation of limit state functions. For this purpose the training data set computed by FEM are needed. A well-chosen number of elements in the training set caused that trained network has good generalization in properties in spite of simple architecture. The biggest error in testing formula not exceeded $1 \%$. These properties indicate good efficiency of the resulting formula. It can thus prohibitively reduce the computation time. This approach is applicable to structural reliability problems with a wide arrange of variations including the number of random variables, the random variable distributions and the performance function.

\section{References}

1. H. O. Madsen, S. Krenk, N. C. Lind, Methods of Structural Safety, Prentice-Hall (1986)

2. R. E. Melchers, Structural Reliability Analysis and Predictions, 2nd Ed., Wiley, (1999)

3. O. Ditlevsen, H.O. Madsen, Structural Reliability Methods, Wiley, (1996)

4. P. Thoft-Christensen, M. J. Baker, Structural Reliability. Theory and its Applications, SpringerVerlag, (1982)

5. G. Augusti, A. Baratta, F. Casciati, Probabilistic Methods in Structural Engineering, Chapman and Hall, (1984)

6. M.E. Harr, Reliability-Based Design in Civil Engineering, McGraw-Hill, (1987)

7. A. S. Nowak, K. R. Collins, Reliability of structure, McGraw-Hill Higher Education, (2000)

8. A. Dudzik, Reliability Assessment of SteelAluminium Lattice Tower, IOP Conference Series: Materials Science and Engineering, 245, 1-9 (2017)

9. U. Radoń, Arch. Civ. Mech. Enge. 15(1), 262-271 (2015)

10. A. Dudzik, U. Radoń, The reliability assessment for steel industrial building, Advances In Mechanics: Theoretical, Computational And Interdisciplinary Issues, 163-166 (2016)

11. A. Dudzik, U. Radoń, ACE, LXI (3), 133-147 (2015)

12. K. Kubicka, U. Radoń, ACE, 4, 141-154 (2015)

13. P. Obara, W. Gilewski, J. Kłosowska, Verification of Tensegrity Properties of Kono Structure and Blur
Building, Xxv Polish - Russian - Slovak Seminar Theoretical Foundation Of Civil Engineering, 153, 173-179 (2016)

14. P. Obara, W. Gilewski, Bull. Pol. Acad. Sci.-Te., 64(4), 739-750 (2016)

15. M.F. Anjum, T. Imran, A.-S. Khaled, Eur. J. Oper. Res., 101, 65-73 (1997)

16. K. Hornik, M. Stinchcombe, H. White, Neural Networks 3, 551-560 (1990)

17. J.H.J. Garrett, J. Comput. Civil. Eng., ASCE 8(2), 129-130 (1994)

18. T. Masters, Practical Neural Network Recipes in $C++$, Academic Press, San Diego (1993)

19. T. Sasaki, A neural network-based response surface approach for computing failure probabilities, In: Corotis, R.B., Schueller, G.I., Shinozuka, M. (Eds.), 8th International Conference on Structural Safety and Reliability (ICOSSAR 2001), USA (2001)

20. A.T.C. Goh, F.H. Kulhawy, Can. Geotech. J., 40(6), 1235-1244 (2003)

21. J. Deng, D.S. Gu, X.B. Li, Z.Q. Yue, Struct. Saf. 25(1), 25-48 (2005)

22. T. Most, Approximation of complex nonlinear functions by means of neural networks, 2nd Weimar Optimization and Stochastic Days (2005)

23. Y. Tsompanakis, N. Lagaros, G. E. Stavroulaki, Efficient Neural Network Models for Structural Reliability Analysis and Identification Problems, The Eighth International Conference on the Application of Artificial Intelligence to Civil, Structural and Environmental Engineering (2005)

24. M. Papadrakakis, V. Papadopoulos, N. Lagaros, Comput. Method. Appl. M., 136, 145-163 (1996)

25. E. Pabisek, J. Kaliszuk, Z. Waszczyszyn, Neural and finite element analysis of a plane steel frame reliability by the Classical Monte Carlo method. Artificial Intelligence and Soft Computing - ICAISC 2004, 7th International Conference, Zakopane, 10811086 (2004)

26. http://numpress.ippt.gov.pl/index.html

27. A.M. Hasofer, N.C. Lind, J. Eng. Mech. Div.-ASCE, 100, 111-121 (1974)

28. D. T. Tracey, C. E. Freese, Comput. Struct., 13, 4553 (1981) 